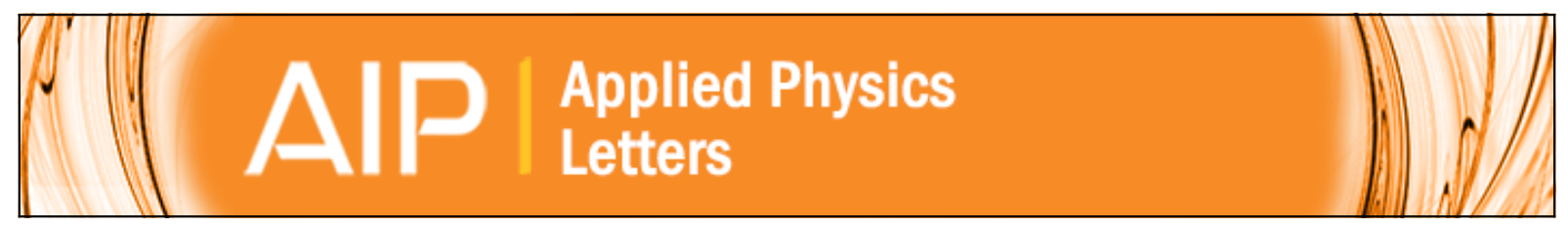

\title{
Vortex-antivortex pair driven magnetization dynamics studied by micromagnetic simulations
}

Ki-Suk Lee, Byoung-Woo Kang, Young-Sang Yu, and Sang-Koog Kim

Citation: Applied Physics Letters 85, 1568 (2004); doi: 10.1063/1.1784892

View online: http://dx.doi.org/10.1063/1.1784892

View Table of Contents: http://scitation.aip.org/content/aip/journal/apl/85/9?ver=pdfcov

Published by the AIP Publishing

\section{Articles you may be interested in}

Limits for the vortex state spin torque oscillator in magnetic nanopillars: Micromagnetic simulations for a thin free layer

J. Appl. Phys. 108, 123914 (2010); 10.1063/1.3524222

Magnetization reversal of $\mathrm{Co} / \mathrm{Cu} / \mathrm{Co}$ elliptical elements studied by in-field magnetic force microscope and micromagnetic simulation

J. Appl. Phys. 103, 093910 (2008); 10.1063/1.2917330

Micromagnetic simulations of nanosecond magnetization reversal processes in magnetic nanopillar J. Appl. Phys. 99, 08G522 (2006); 10.1063/1.2177049

Micromagnetic simulation studies of ferromagnetic part spheres

J. Appl. Phys. 97, 10E305 (2005); 10.1063/1.1850073

Vortex-antivortex assisted magnetization dynamics in a semi-continuous thin-film model system studied by micromagnetic simulations

Appl. Phys. Lett. 86, 052504 (2005); 10.1063/1.1855413

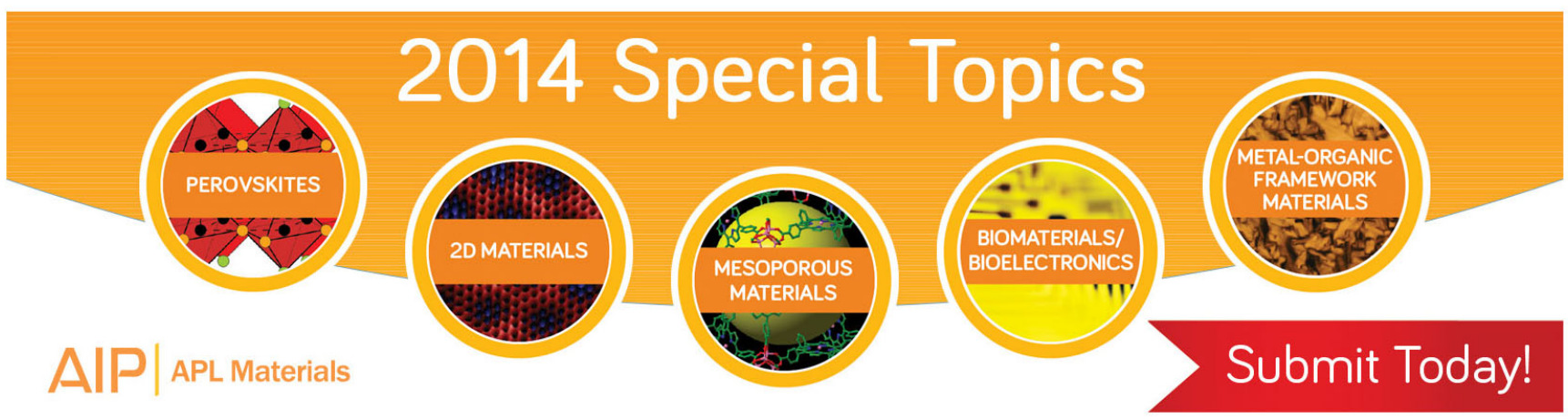




\title{
Vortex-antivortex pair driven magnetization dynamics studied by micromagnetic simulations
}

\author{
Ki-Suk Lee, Byoung-Woo Kang, ${ }^{\text {a) }}$ Young-Sang Yu, and Sang-Koog Kim ${ }^{\text {b) }}$ \\ Nanospintronics Laboratory, School of Materials Science and Engineering, and Research Institute of \\ Advanced Materials, College of Engineering, Seoul National University, Seoul 151-744, Korea
}

(Received 9 April 2004; accepted 22 June 2004)

\begin{abstract}
Magnetization dynamics approaching an equilibrium vortex state from an initial nonequilibrium state under zero magnetic field has been studied in a circular-shaped Fe disk with a thickness of $5 \mathrm{~nm}$ and a diameter of $1200 \mathrm{~nm}$ using micromagnetic simulations. Upon starting from the initial random configuration of in-plane magnetizations, a great number of vortex $(V)$-antivortex $(\bar{V})$ pairs are generated at a lot of nucleation sites where both types of $V$ and $\bar{V}$ are energetically favorable to form. The $V$ and $\bar{V}$ are propagated and then annihilated by their attractive interactions during the relaxation dynamic process. These results reveal that temporal magnetization evolutions can be dominated by the nucleation of $V-\bar{V}$ pairs, followed by their propagation and annihilation. The dynamic process driven by $V-\bar{V}$ pairs can play a significant role in various magnetization reversals. () 2004 American Institute of Physics. [DOI: 10.1063/1.1784892]
\end{abstract}

The so-called magnetic vortex has a curling structure of magnetization $(\mathbf{M})$ and a perpendicular component $\left(M_{z}\right)$ at its core $^{1}$ in circular, rectangular, ellipse, and stadium-shaped microfabricated elements of ultrathin magnetic films. This type vortex $(V)$ has attracted much attention ${ }^{2-8}$ because of both its fundamental interest and technological applications to ultrathin-film magnetic memory devices. The internal structure of such vortex states in micron-sized elements comparable to the length scale of magnetostatic interactions have been verified by magnetic force microcopy, ${ }^{4}$ Lorentz transmission electron microcopy, ${ }^{5}$ and spin-polarized scanning tunneling microscopy ${ }^{6}$ with a nanometer-scale (or less) spatial resolution. Furthermore, experimental studies not only on the magnetic vortices, ${ }^{3}$ but also their interactions in magnetic-field driven $\mathbf{M}$ reversals ${ }^{7}$ have been intensively carried out with confined systems such as patterned dots or their arrays. In addition, analytical interpretations ${ }^{8,9}$ and theoretical micromagnetic simulations ${ }^{10-12}$ of the characteristic structures and the dynamical process of vortices ${ }^{9}$ in such elements have provided insights into the fundamental understanding of their related static or dynamic properties in various $\mathbf{M}$ reversals. ${ }^{13}$

On the other hand, the counterpart of a type of $V$, i.e., antivortex $(\bar{V})$ which has cross-bloch lines around its core of either up or down orientation of $M_{z}$, has been usually found in the periodic arrangement of alternating $V$ and $\bar{V}$ on a cross-tie wall at saw-tooth $180^{\circ}$ domain boundaries in ultrathin magnetic films. ${ }^{1}$ In particular, $V-\bar{V}$ pairs were also observed at the forefront of small needle-shaped domains growing into oppositely oriented $180^{\circ}$ domains in a continuous 33-nm-thick Fe film by using scanning transmission $\mathrm{x}$-ray microscopy. ${ }^{14}$ Quite recently, Eames and Dahlberg, ${ }^{15}$ and Okuno et al. ${ }^{16}$ also reported the experimental observations of

\footnotetext{
${ }^{a}$ Also at: Department of Materials Science and Engineering, Massachusetts Institute of Technology, 77 Massachusetts Avenue, Cambridge, Massachusetts 02139 .

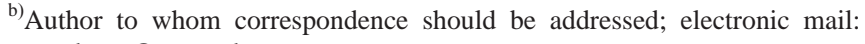
sangkoog@snu.ac.kr
}

$\bar{V}$ as well as $V$ in submicron stadium-shaped permalloy films and have implicated both types of $V$ and $\bar{V}$ in magnetic-field driven $\mathbf{M}$ reversals. However, the roles of the $V-\bar{V}$ pairs in $\mathbf{M}$ evolution dynamics have not been studied in detail in terms of their nucleation, propagation, and annihilation by considering their attractive interactions.

In this letter, we carry out micromagnetic simulations on an $\mathbf{M}$ dynamic process approaching the equilibrium vortex state from an initial nonequilibrium state of random in-plane $\mathbf{M}$ orientation under zero magnetic field in a model system of a circular shaped Fe disk. The dynamic process associated with many $V-\bar{V}$ pairs is discussed in terms of their nucleation, propagation, and annihilation that are driven by the attractive interactions between the different types of $V$ and $\bar{V}$.

Micromagnetic simulations were carried out on $\mathbf{M}$ dynamics in a circular shaped $\mathrm{Fe}$ disk having a thickness of $5 \mathrm{~nm}$ and a diameter of $1200 \mathrm{~nm}$ using the object-oriented micromagnetic framework (OOMMF). ${ }^{17}$ The physical parameters for individual cubic cells of a $5 \times 5 \times 5 \mathrm{~nm}^{3}$ dimension are used as follows: an exchange constant of $A=4.2$ $\times 10^{-11}(\mathrm{~J} / \mathrm{m})$, a saturation magnetization of $M_{s}=1.7$ $\times 10^{6}(\mathrm{~A} / \mathrm{m})$, an exchange length of $L_{\mathrm{ex}}=4.8 \mathrm{~nm}$, an anisotropy constant of $K=0$, and a damping parameter of $\alpha=0.5 .^{18}$

Figure 1 shows a magnetization vector image on many interesting features of microstructures taken at a time of $t$ $=0.59 \mathrm{~ns}$ during the relaxation process under zero magnetic field as mentioned earlier. White or black small spots indicate up or down orientation of $M_{z}$ that is largely localized at the cores of both types of $V$ and $\bar{V}$. Circular- and cross-bloch lines around each core represent circular- and cross-type vortices, respectively. The cross-type vortex is called $\bar{V}$ to distinguish it from the counterpart circular $V$. The $V$ has energetically equivalent four states of $V_{R}^{\uparrow}, V_{L}^{\uparrow}, V_{R}^{\downarrow}, V_{L}^{\downarrow}$ with left $(L)$ or right $(R)$ handedness with up $(\uparrow)$ or down $(\downarrow)$ core orientation. In contrast, the $\bar{V}$ has only two states of $\bar{V}^{\uparrow}$ and $\bar{V} \downarrow$ that are characterized by either of the up and down core orientations, as shown in Fig. 1. The characteristic in-plane orien- 


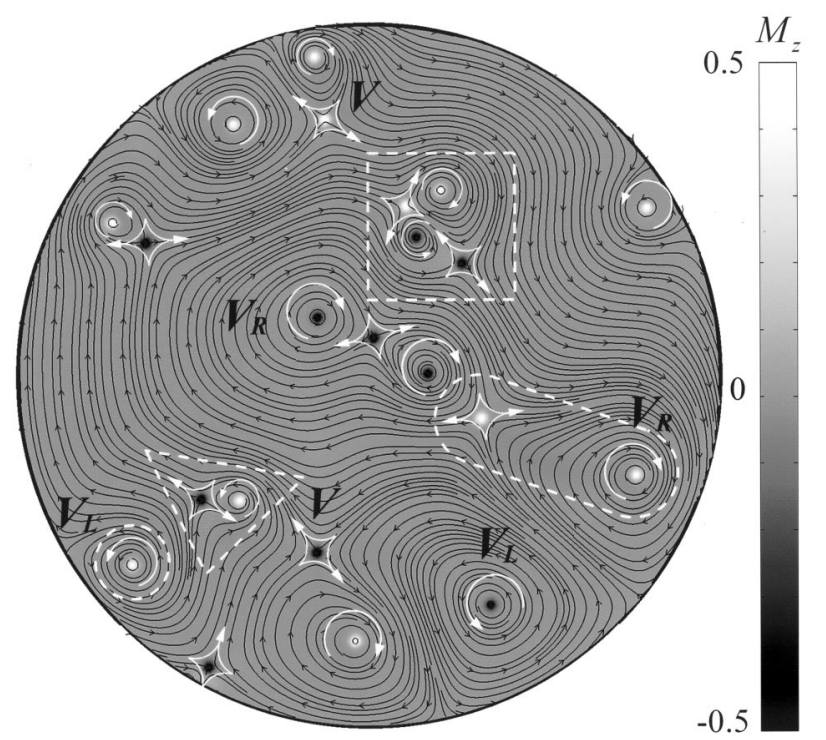

FIG. 1. The plane-view image of magnetic microstructures taken at $t$ $=0.59 \mathrm{~ns}$ during a relaxation dynamics approaching the equilibrium vortex state from an initial random in-plane $\mathbf{M}$. The gray scale indicates the $M_{z}$ component, while the contour lines with small arrows represent the in-plane directions of $\mathbf{M}$. The white and black spots represent up and down core orientations of both types of $V$ and $\bar{V}$. The characteristic structures of various vortex states are denoted by symbols as noted, which are described in the text. Dotted-lines of square, triangle, stadium, and circle shapes highlight the various features of vortices interacting with each other.

tation of $\bar{V}$ is determined by the winding in-plane $\mathbf{M}$ of its neighboring counterpart $V$. Many striking features regarding $V$ and $\bar{V}$ are found as follows: (1) An array of $V$ and $\bar{V}$ being similar to a segment of the cross-tie wall, (2) $V-\bar{V}$ pairs, and (3) apparently isolated $V$ and $\bar{V}$. It is certain from the results that the $V-\bar{V}$ pairs and $V$ or $\bar{V}$ are related closely to $\mathbf{M}$ dynamic process.

To clarify the role of both types of $V$ and $\bar{V}$ in dynamic $\mathbf{M}$ evolution, the temporal evolution of magnetic microstructures is shown in Fig. 2, starting from an initial random configuration of the in-plane $\mathbf{M}(t=0 \mathrm{~s})$ toward its final equilibrium vortex state under zero magnetic field. We also plot the numbers of $V$ and $\bar{V}$, the average of $M_{z}^{2}$ over individual cells $\left(\left\langle M_{z}^{2}\right\rangle\right)$ and exchange $\left(E_{\mathrm{ex}}\right)$ and magnetostatic $\left(E_{\mathrm{ms}}\right)$ en-

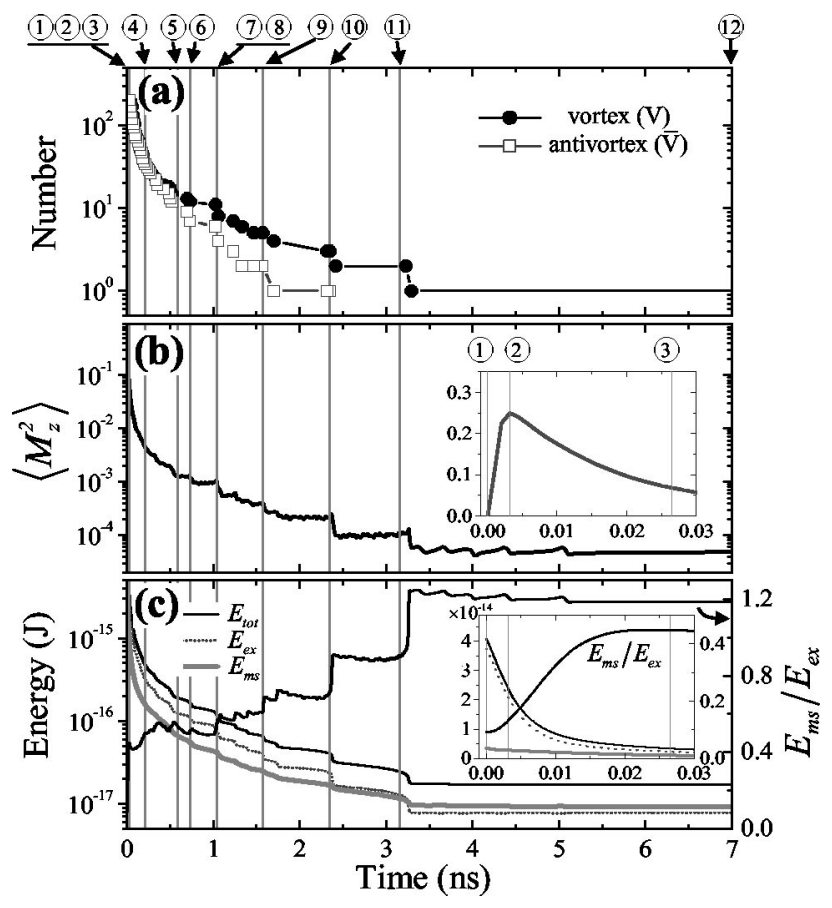

FIG. 3. Time dependence of the numbers of $V$ and $\bar{V}$ in (a), $\left\langle M_{z}^{2}\right\rangle$ in (b), and $E_{\mathrm{ex}}, E_{\mathrm{ms}}$, and $E_{\mathrm{tot}}$ in (c). The vertical lines with the corresponding numbers are marked for each image shown in Fig. 2. The insets in (b) and (c) show the variations of $\left\langle M_{z}^{2}\right\rangle$ and $E$ in the early time of the relaxation dynamics.

ergies, and the sum $\left(E_{\text {tot }}\right)$ of the two energy terms as a function of $t$ in Fig. 3. The in-plane random orientation at $t$ $=0 \mathrm{~s}$ is represented by $\left\langle M_{z}^{2}\right\rangle=0$ together with the largest values of $E_{\mathrm{ex}}$ and $E_{\mathrm{ms}}$ (state (1). As soon as the relaxation process starts, the $\left\langle M_{z}^{2}\right\rangle$ value reaches a maximum to reduce the largest $E_{\mathrm{ex}}$ for the random in-plane orientation (state (2)). To reduce $E_{\text {ex }}$ more, a great number of $V$ and $\bar{V}$ start to nucleate at available nucleation sites where they are energetically favorable to form (state (3)). Well-formed $V$ and $\bar{V}$ appear at $t$ $=0.21 \mathrm{~ns}$ (state (4)) to decrease $E_{\mathrm{ex}}$ as well as $E_{\mathrm{ms}}$, and then they start to propagate and annihilate as a further relaxation proceeds.

Specifically, we highlight interactions between $V$ and $\bar{V}$ present in the areas indicated by dotted-line square, circle, stadium, and triangle, starting from $t=0.59 \mathrm{~ns}$ (state (5)). The

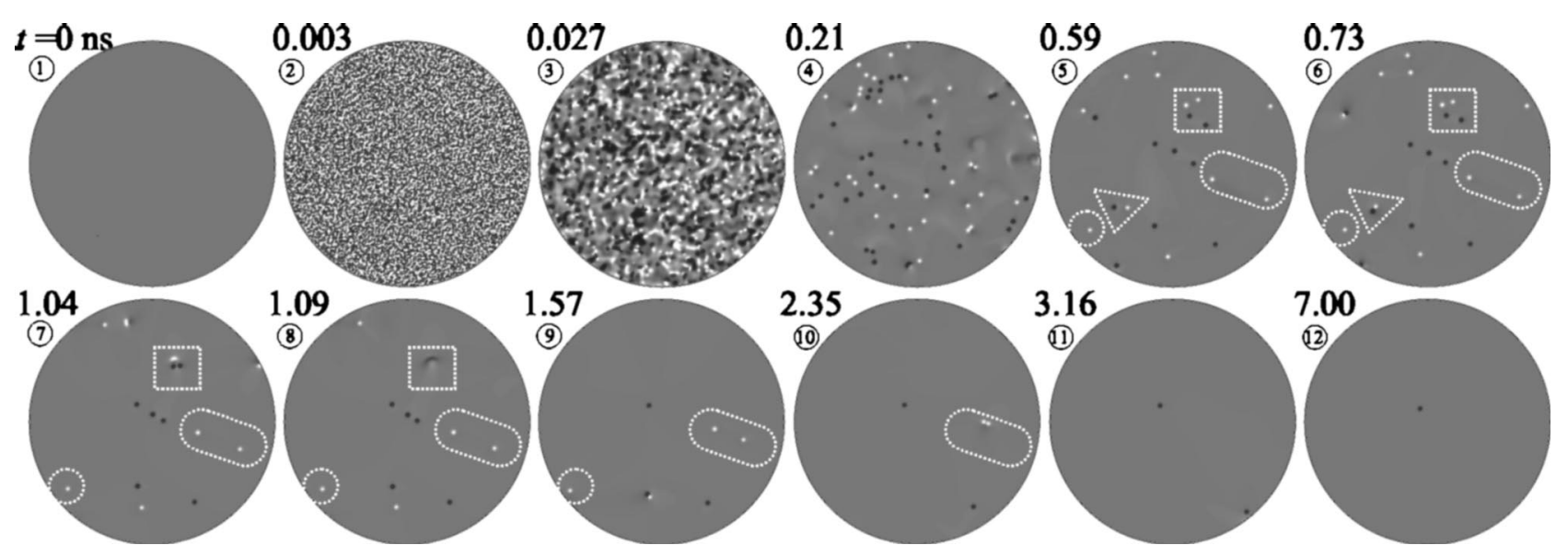

FIG. 2. The temporal evolution of magnetic microstructures taken at each time as noted during the relaxation dynamic process. The gray scale represents the $M$ component. The white and black spots indicate up and down orientations of both $V$ and $\bar{V}$ cores. The dotted-lines of triangle, square, stadium, and circle highlight striking features of interactions between various vortex structures. 

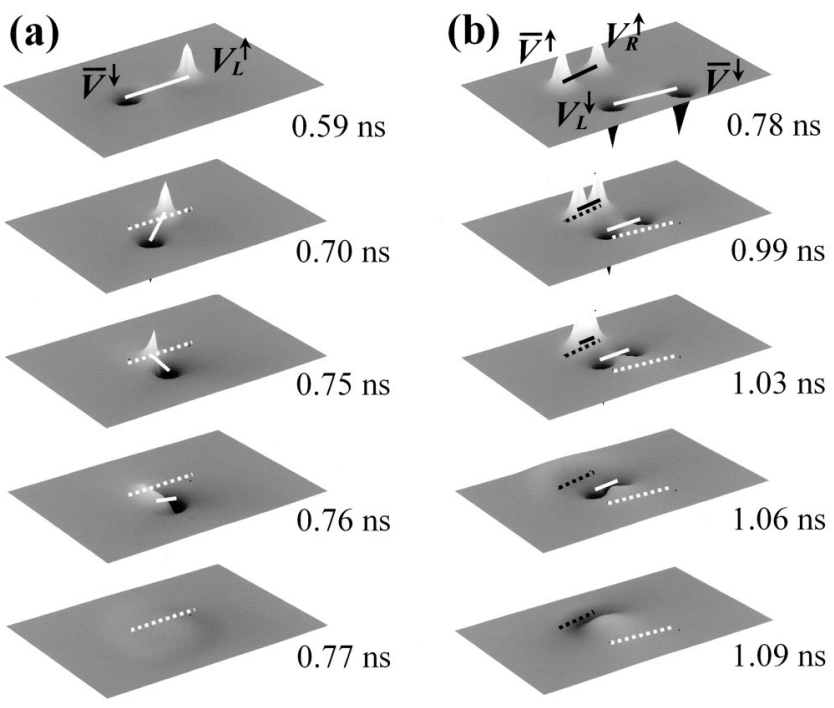

FIG. 4. Perspective views of the propagation and annihilation processes of $V-\bar{V}$ pairs present in the areas noted by the dotted-line triangles and squares shown in Fig. 2. The dashed and solid lines indicate axes connecting between each core of $V$ and $\bar{V}$ that propagate and annihilate as the relaxation process proceeds.

$V_{L}^{\uparrow}$ and $\bar{V} \downarrow$ in the triangle-marked area disappear through their propagation and annihilation processes $(5 \rightarrow(6) \rightarrow(7)$ ). The four different vortices in the square-marked area also disappear through the attractive forces between $V_{R}^{\uparrow}$ and $\bar{V} \uparrow$, and $V_{L}^{\downarrow}$ and $\bar{V}^{\downarrow}$, while seemingly isolated $V_{L}^{\uparrow}$ in the dotted-line circle is slowly taken out through the boundary. As shown in the stadium marked area, it is noticeably interesting that $\bar{V}^{\uparrow}$ attracts the counterpart $V_{R}^{\uparrow}$ placed near the boundary of the disk, although the two are far away from each other. As the propagation proceeds further, $E_{\mathrm{ex}}, E_{\mathrm{ms}}$, and its ratio $\left(E_{\mathrm{ms}} / E_{\mathrm{ex}}\right)$ gradually decrease, while $E_{\mathrm{ex}}, E_{\mathrm{ms}}$, and the inverse of $E_{\mathrm{ms}} / E_{\mathrm{ex}}$ suddenly decrease upon the annihilation of each of $V-\bar{V}$ pairs. ${ }^{19}$ Consequently, attractive interactions between each counterpart vortex lead to their propagation and annihilation, thus governing the $\mathbf{M}$ dynamic process triggered by a great number of $V$ and $\bar{V}$ generated at the earlier stages.

Finally, detailed features of the interactions between $V_{L}^{\uparrow}$ and $\bar{V} \downarrow$ are shown in Fig. 4(a), and $V_{R}^{\uparrow}$ and $\bar{V}^{\uparrow}, V_{L}^{\downarrow}$ and $\bar{V} \downarrow$ in Fig. 4(b). This figure clearly shows that core-core distances in each pair decrease and then individual $V$ and $\bar{V}$ collapse by their attractive interactions as the relaxation process proceeds. Also, the pair axis connecting each core of the $V-\bar{V}$ pair with their opposite core orientations rotates in the plane by nearly $180^{\circ}$ due to involved torques [Fig. 4(a)]. On the other hand, such rotation is negligible for the same core ori- entation, but still occurs somewhat due to the interactions with the neighboring vortices as shown in Fig. 4(b). These results indicate that $V$ and $\bar{V}$, and their interactions can play an important role in dynamic $\mathbf{M}$ evolutions.

In conclusion, $\mathbf{M}$ dynamics can be dominated by the nucleation process of $V-\bar{V}$ pairs, followed by their propagation and annihilation driven by the attractive interactions between different types of $V$ and $\bar{V}$. The $V-\bar{V}$ pairs play a crucial role in $\mathbf{M}$ dynamic process, and thus the $V-\bar{V}$ pair driven $\mathbf{M}$ evolution should be considered in the understanding of static or dynamic $\mathbf{M}$ reversals.

The authors acknowledge K.-J. Lee for his valuable discussions. This work was supported by the Korea Science and Engineering Foundation (KOSEF) through q-Psi at Hanyang University.

${ }^{1}$ A. Hubert and R. Schäfer, Magnetic Domains (Springer, Berlin, 1998).

${ }^{2}$ M. Hehn, K. Ounadjela, J.-P. Bucher, F. Rousseaux, D. Decanini, B. Bartenlian, and C. Chappert, Science, 272, 1782 (1996).

${ }^{3}$ R. P. Cowburn, D. K. Koltsov, A. O. Adeyeye, M. E. Welland, and D. M. Tricker, Phys. Rev. Lett. 83, 1042 (1999).

${ }^{4}$ T. Shinjo, T. Okuno, R. Hassdorf, K. Shigeto, and T. Ono, Science 289, 930 (2000).

${ }^{5}$ J. Raabe, R. Pulwey, R. Sattler, T. Schweinböck, J. Zweck, and D. Weiss, J. Appl. Phys. 88, 4437 (2000).

${ }^{6}$ A. Wachowiak, J. Wiebe, M. Bode, O. Pietzsch, M. Morgenstern, and R. Wiesendanger, Science 298, 577 (2002).

${ }^{7}$ R. P. Cowburn, A. O. Adeyeye, and M. E. Welland, New J. Phys. 1, 16 (1999).

${ }^{8}$ K. Yu. Guslienko, V. Novosad, Y. Otani, H. Shima, and K. Fukamichi,Phys. Rev. B 65, 024414 (2001).

${ }^{9}$ K. Yu. Guslienko, V. Novosad, Y. Otani, H. Shima, and K. Fukamichi, Appl. Phys. Lett. 78, 3848 (2001).

${ }^{10}$ J. K. Ha, R. Hertel, and J. Kirschner, Phys. Rev. B 67, 224432 (2003).

${ }^{11}$ N. Dao, S. L. Whittenburg, and R. P. Cowburn, J. Appl. Phys. 90, 5235 (2001).

${ }^{12}$ R. Hertel and J. Kirschner, J. Magn. Magn. Mater. 270, 364 (2004).

${ }^{13}$ S.-B. Choe, Y. Acremann, A. Scholl, A. Bauer, A. Doran, J. Stöhr, and H. A. Padmore, Science 304, 420 (2004).

${ }^{14}$ S.-K. Kim, J. B. Kortright, and S.-C. Shin, Appl. Phys. Lett. 78, 2742 (2001).

${ }^{15}$ P. Eames and E. D. Dahlberg (unpublished).

${ }^{16}$ T. Okuno, K. Mibu, and T. Shinjo, J. Appl. Phys. 95, 3612 (2004).

${ }^{17}$ For details, see http://math/nist.gov/oommf.

${ }^{18}$ This large value is used to save computation time for micromagnetic simulations, although a much lower value is suitable for investigating such picotime-scale dynamic process. We also performed it with $\alpha=0.01$ and confirmed that the resultant microstructures on $V$ and $\bar{V}$ remain similar to those calculated using $\alpha=0.5$, except for the occurrence and propagation of magnetization waves starting at a position where $V$ and $\bar{V}$ collapse, as also found in Ref. 12. Further studies on the spin waves will be carried out

${ }^{19}$ Also, the values of $E_{\mathrm{ex}}, E_{\mathrm{ms}}$, and $E_{\mathrm{ex}} / E_{\mathrm{ms}}$ abruptly decrease when $V$ or $\bar{V}$ is taken out through the boundary. 